\title{
Carcinoma de pulmón de origen laboral
}

\section{Occupational lung cancer}

\section{Pérez de las Casas, B. Fernández Infante}

\section{RESUMEN}

El carcinoma broncopulmonar es la primera causa de muerte por cáncer en varones, siendo su principal causa el consumo de tabaco; no obstante, diversos estudios han atribuido un tanto por ciento no despreciable de su etiología a la exposición laboral a agentes considerados cancerígenos como el asbesto, con el que tiene relación la mitad de los casos de cáncer pulmonar de origen laboral. Dada la baja supervivencia de esta patología, son de suma importancia las medidas de prevención encaminadas a identificar los agentes cancerígenos y a la disminución de su exposición. Puesto que la presentación clínica no difiere del carcinoma relacionado con el tabaco, un alto grado de sospecha, basado en una cuidadosa historia laboral, es fundamental para su diagnóstico. Debido el efecto sinérgico del tabaco, medidas destinadas a disminuir su consumo, continúan siendo de suma importancia en la población expuesta.

Palabras clave. Cáncer de pulmón. Enfermedad ocupacional. Asbesto. Radón. Sílice. Arsénico. Hidrocarburos aromáticos policíclicos.

\begin{abstract}
Bronchopulmonary carcinoma is the first cause of death by cancer in males, its principal cause being tobacco consumption. Nonetheless, different studies have attributed a certain, by no means negligible percent of its aetiology to the occupational exposure to agents considered carcinogenic such as asbestos, with which half of the cases of occupational lung cancer are related. Given the low survival rate of this pathology, preventive measures directed at identifying carcinogenic agents and reducing exposure to them are extremely important. Given that the clinical presentation does not differ from tobacco-related carcinoma, a high level of suspicion, based on a meticulous occupational history, is fundamental to its diagnosis. Due to the synergic effect of tobacco, measures aimed at reducing its consumption continue to be extremely important in the exposed population.
\end{abstract}

Key words. Lung cancer. Occupational disease. Asbestos. Radon. Silica. Arsenic. Polycyclical aromatic hydrocarbons.

An. Sist. Sanit. Navar. 2005; 28 (Supl. 1): 101-106.

Sección de Neumología. Hospital de Navarra. Pamplona.

\section{Correspondencia:}

Manuel Pérez de las Casas

Sección de Neumología

Hospital de Navarra

Irunlarrea, 3

31008 Pamplona

Tfno. 848422288

e-mail: mperezde@cfnavarra.es 


\section{INTRODUCCIÓN}

El cáncer de pulmón es el tumor más frecuente y el que da lugar a una mayor mortalidad, tanto a nivel mundial como en nuestro país, con una incidencia en 1990 de 51,6 y 3,4 casos por cada 100.000 en hombres y mujeres, respectivamente ${ }^{1}$, siendo la supervivencia a los 5 años no mayor del $15 \%$.

No cabe duda de que el consumo de tabaco es la principal causa del cáncer de pulmón, pero hay que tener en cuenta la existencia de otros factores de riesgo como la exposición a carcinógenos en el medio laboral.

\section{FACTORES OCUPACIONALES}

No resulta fácil saber el papel que los diferentes carcinógenos presentes en el medio laboral juegan en el desarrollo del carcinoma bronquial debido a la interacción de los mismos, la existencia simultánea de otros factores de riesgo conocidos como el tabaco, el periodo transcurrido entre la exposición y el desarrollo de la enfermedad y la falta de información sobre las sustancias a las que los trabajadores están expuestos.

Doll y Peto $^{2}$ estimaron a comienzos de los años 80 que el 15\% de los casos de cáncer pulmonar en varones y el $5 \%$ en mujeres tenían relación con factores ocupacionales, cifras refrendadas posteriormente por otros autores ${ }^{3,4}$.
En nuestro país, según la base de datos CAREX (CARcinogen EXposure), se estima que entre 1990 y 1993 había 3,1 millones de trabajadores (25\% de los empleados) expuestos a sustancias reconocidas como carcinógenos. El mayor número correspondía a la sílice cristalina (400.000), humos de motor diesel (275.000), radón (280.000) y benceno (90.000). Según García y Kogevinas ${ }^{5}$, en 1991 un total de 3.011 muertes por cáncer en varones y 272 en mujeres fueron atribuibles a factores laborales, correspondiendo el $65 \%$ de las ocurridas en varones a cáncer de pulmón. El porcentaje total de muertes por cáncer atribuidas a exposición laboral es del $4 \%$.

La IARC (Internacional Agency for Research on Cancer), dependiente de la Organización Mundial de la Salud, es la encargada de analizar las evidencias epidemiológicas y experimentales que llevan a declarar a una sustancia como carcinógeno, clasificando a éstas dentro de cinco grupos:

- Grupo 1: carcinógeno probado.

- Grupo 2 A: carcinógeno probable.

- Grupo 2 B: carcinógeno posible.

- Grupo 3: no clasificable como carcinógeno en humanos.

- Grupo 4: probablemente no carcinógeno.

Por razones de espacio, se exponen algunos de los incluidos en el grupo 1 y 2 A, remitiendo al lector a la dirección electrónica de la citada agencia (www.IARC.fr) para mayor información (Tabla 1).

Tabla 1. Agentes cancerígenos.

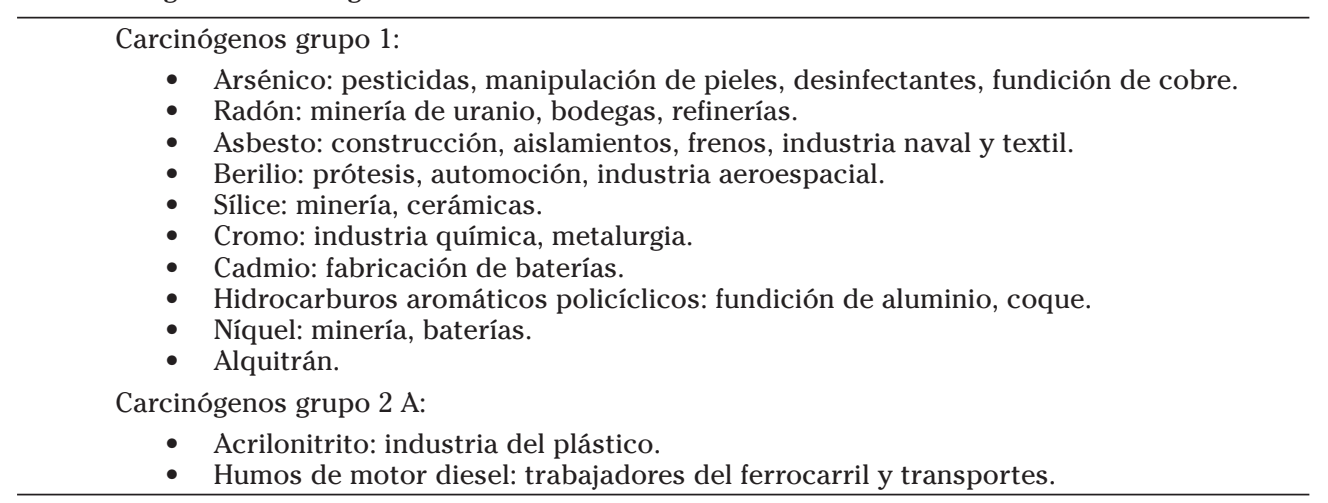




\section{EVALUACIÓN CLÍNICA}

El carcinoma bronquial de origen laboral no difiere, en cuanto a la presentación clínica o patológica del originado por otras causas. Sin embargo, establecer su relación con el lugar de trabajo tiene repercusiones, tanto a nivel de salud pública (permite adoptar medidas preventivas) como de forma individual (posibilidad de indemnizaciones) ${ }^{6}$.

La clave para el reconocimiento de un caso de cáncer de pulmón de origen laboral es un alto grado de sospecha clínica. Se debe realizar una completa historia laboral, de manera que la integración de la misma junto con el antecedente tabáquico, la latencia desde la exposición y la inclusión del agente sospechado en las categorías propuestas por la IARC nos permitan formar una opinión sobre la etiología del proceso tumoral.

A continuación se exponen algunos de los carcinógenos conocidos y su relación con el carcinoma bronquial.

\section{ASBESTO}

El asbesto está entre los más importantes carcinógenos humanos. Se estima que entre 1940 y 1980, 27 millones de personas estuvieron potencialmente expuestas al mismo en EEUU, de las cuales 18 millones tuvieron exposiciones excesivas equivalentes a 2 meses de trabajo en manufactura primaria de asbesto ${ }^{7}$.

En EEUU, de los 10.000 casos anuales de cáncer de pulmón de origen laboral, se cree que la mitad son secundarios a amianto $^{8}$, siendo el cáncer de pulmón el principal cáncer relacionado con este agente, constituyendo el $20 \%$ de las muertes de los expuestos ${ }^{9,10}$. Al menos el $4 \%$ de todos los cánceres es atribuible a la exposición a asbesto ${ }^{10}$.

En términos generales, se piensa que en España el asbesto es el carcinógeno ocupacional más importante ${ }^{5}$. Para el pulmón es también, entre todos los agentes potencialmente tóxicos, el que tiene mayor importancia, habiéndose referido también cifras de hasta el $20 \%$ de fallecimientos por cáncer de pulmón en algunas poblaciones expuestas ${ }^{3,11}$.
Tanto en estudios realizados en otros países $^{12,13}$, como en España ${ }^{14,15}$, las profesiones que conllevan exposición al asbesto (construcción, talleres, astilleros, etc.) han estado habitualmente entre las más asociadas con el cáncer de pulmón de origen laboral.

En la etiopatogenia de la enfermedad probablemente juegue un papel la producción de radicales libres y, como consecuencia, el daño del $\mathrm{ADN}^{16,17}$. La delección homocigota del gen de la glutatión S-transferasa M1 (GSTM1), o el genotipo acetilador lento de $\mathrm{N}$-acetiltransferasa 2 (NAT2), pueden conferir un riesgo adicional ${ }^{18}$.

El riesgo de desarrollar cáncer de pulmón es el doble en personas expuestas respecto de las no expuestas ${ }^{8}$. La diferencia de riesgo entre unas industrias y otras (bajo en minería y alto en textil) sería debido a las diferentes características de las fibras, habiéndose establecido una relación dosis-respuesta, existiendo controversia en cuanto a si dosis bajas de exposición pueden producir cáncer de pulmón o si existe un umbral de exposición ${ }^{19-21}$.

Aunque la mayoría de los cánceres inducidos por asbesto se dan en individuos con asbestosis, se acepta en general que ésta no es un requisito para atribuir el cáncer a la exposición al asbesto ${ }^{22}$. En la revisión efectuada por Steenland ${ }^{8}$, la asbestosis tuvo un riesgo relativo de 5,91 (95\% IC, 4,98-7,00).

Existen trabajos que sugieren un efecto sinérgico entre tabaco y asbesto, circunstancia ésta que tiene una mayor transcendencia en países como España, donde tanto la prevalencia de tabaquismo como la exposición al asbesto han sido elevadas en las últimas décadas, aunque afortunadamente tienden a reducirse $\mathrm{e}^{5,23-24}$. En expuestos fumadores el riesgo es 60 veces mayor que en no fumadores no expuestos $^{25}$. En no fumadores expuestos existen trabajos que atribuyen un riesgo relativo de 1,1 a $15,0^{26}$.

En cuanto a la estirpe celular, unos investigadores reportan más casos de adenocarcinoma en expuestos ${ }^{27}$, y otros no encuentran diferencias entre cánceres debidos al tabaco y los secundarios al asbesto $^{28}$. La combinación de tabaco y 
asbesto se asocia con una localización del tumor en lóbulos superiores ${ }^{29}$; si no se da esta combinación, unos trabajos encuentran predominancia en lóbulos inferiores ${ }^{30}$, mientras en otros no es concluyente ${ }^{31}$.

Establecer una historia de exposición que comience al menos 10 años antes del diagnóstico es de ayuda para determinar la causa del tumor, así como identificar indicadores de severidad de la exposición (como placas pleurales o gran número de cuerpos de asbesto o abundantes fibras de asbesto en el tejido pulmonar resecado ${ }^{32}$.

\section{RADÓN}

El radón es un gas inerte, inodoro e incoloro, ubicuo, procedente de la desintegración natural del radio, el cual a su vez es producto de la desintegración del uranio, presente en gran parte del suelo y de las rocas de la corteza terrestre, que se acumula con facilidad en lugares cerrados. Entre los trabajos con exposición a radón se pueden citar los desarrollados en minas subterráneas (uranio, vanadio), bodegas y cuevas naturales, refinerías de petróleo, balnearios con aguas radiactivas o túneles.

\section{Radón en domicilios}

En algunos locales mal ventilados se han llegado a detectar niveles de inhalación similares a los observados en las minas de uranio, por lo que se considera la propia casa como una importante fuente de exposición. En este caso, el radón se produce por la fisión espontánea del uranio 238 emanado del vinculado por la conducción de agua. El radón y sus productos de desintegración (polonio 218, 214 y 210) emiten partículas radiactivas con un tamaño entre 40 y 70 micrones. Estas partículas alfa se unen al polvo y son inhaladas dañando el núcleo de las células basales del epitelio bronquial, donde se inicia la carcinogénesis $^{33}$.

En un metaanálisis realizado en 1997 se encontró que las personas expuestas en el domicilio a unas concentraciones de radón de 4 picocurios por litro de aire (pCi/l) tenían un riesgo de desarrollar el tumor discretamente superior al de las personas no expuestas (RR: 1,14; IC: 1,0$1,3)^{34}$. La tendencia observada en el estu- dio entre riesgo y exposición fue similar a las extrapolaciones realizadas en mineros que habían tenido en su trabajo un nivel de exposición equivalente al de los domicilios, lo que apoya el uso de un modelo de riesgo lineal para la evaluación de la patología asociada al radón residencial ${ }^{35}$.

\section{SÍLICE}

Aunque la sílice cristalina ha sido introducida como agente carcinógeno tipo 1 en la última clasificación de la IARC, su inclusión continúa siendo motivo de polémica. Algunos estudios en los que se basa esta inclusión presentan defectos metodológicos serios, y sus resultados son dispares. Unos autores muestran la relación entre silicosis y cáncer; según otros, esta relación aparece con la presencia de adenopatías sin alteración parenquimatosa, o con el tiempo de exposición a sílice en ausencia de silicosis. Si bien los estudios realizados más recientemente muestran un mejor diseño y control de los sesgos, posiblemente se necesiten más datos sobre los polimorfismos genéticos y la exposición para confirmar, de una manera definitiva, la relación entre inhalación de sílice y cáncer de pulmón ${ }^{36}$.

\section{ARSÉNICO}

Las industrias con exposición a arsénico incluyen minas de metales, trabajo en viñas, producción de pesticidas de arsénico y sus aplicaciones, fundición de cobre y manufacturas de pieles.

Abundantes estudios epidemiológicos en plantas de pesticidas, fundiciones de cobre y entre mineros de estaño en los pasados 40 años han mostrado un riesgo aumentado para desarrollar cáncer de pulmón con ratios de mortalidad estandarizada de 1,31 a 5,69, con una clara relación dosis-respuesta ${ }^{3742}$.

El período de latencia es de 25 años, con un rango de 10 a 56 años, teniendo el tabaco un efecto sinérgico ${ }^{43}$.

\section{HIDROCARBUROS AROMÁTICOS POLICÍCLICOS}

La exposición laboral a estos compuestos se da en diferentes ocupaciones, fun- 
damentalmente en los procesos de gasificación del carbón, producción de acero y extracción de petróleo, como producto de la pirólisis de material orgánico, y se ha observado un aumento de neoplasia broncopulmonar en estos trabajadores. El efecto carcinógeno de los humos de los motores diesel estaría en relación con la presencia de estos hidrocarburos y afectaría a los trabajadores del transporte y del ferrocarril $^{6}$, con un riesgo de $2,31^{44}$.

\section{BIBLIOGRAFÍA}

1. Miñarro R, Black RJ, Martínez C, Navarro C, GaRAu I, IZARZugaza I et al. Incidencia y mortalidad por cáncer en España. Patrones y tendencias. Lyon: Internacional Agency for Research on Cancer 2000. Technical Report № 36 .

2. Doll R, Peto R. The causes of cancer: quantitative estimates of avoidable risks of cancer in the United States today. J Natl Cancer Inst 1981; 66: 1191-1308.

3. VAN ZANDWIJK N. Aetiology and prevention of lung cancer. Eur Respir Mon 1995; 1: 18-49.

4. LANGe P, Vestbo J. Lung cancer. Eur Respir Mon 2000; 15: 92-104.

5. García M, Kogevinas M. Estimación de la mortalidad por cáncer laboral y de la exposición a cancerígenos en el lugar de trabajo en España en los años 90. Gac Sanit 1996; 10: 143-151.

6. Martínez GonzÁLEZ C. Neoplasia pulmonar asociada a la exposición laboral. Arch Bronconeumol 2003; 39 (Suppl 4): 15-17.

7. Nicholson WJ, Perkel G, Selikolf IJ. Occupational exposure to asbestos: population at risk and projected mortality1980-2030. Am J Ind Med 1982; 3: 259-311.

8. Steenland K, Loomis D, Shy C, Simonsen N. Review of occupational lung carcinogens.Am J Ind Med 1996, 29: 474-490.

9. LEMEN RA, Dement JM, WAGoner JK. Epidemiology of asbestos-related diseases. Environ Health Perspect 1980, 34: 1-11.

10. Rugo HS, Fischman ML. Occupational cancer. En Occupational and Environmental Medicine. Edited by LaDou J. Stamford, CT: Appletan \& Lange; 1997: 235-271.

11. Takkouche B, Gestal-Otero JJ. The epidemiology of lung cancer: Review of risk factors and spanish data. Eur J Epidemiol 1996; 12: 341-349.

12. Jockel KH, Ahrens W, Jahn I, Pohlabeln H, BOLM-AUDORFF U. Occupational risk factors for lung cancer: a case-control study in West Germany. Int J Epidemiol 1998; 27: 549-560.

13. Pezzoto SM, Poletto L. Occupation and histopatology of lung cancer: a case-control study in Rosario Argentina. Am J Ind Med 1999; 36: 437-443.

14. SÁnchez de Cos J, Riesco JA, Antón J, Díaz SANTAMARÍA P, Márquez PÉrez L, MEdina JF et al. Incidencia del carcinoma broncopulmonar en Extremadura durante el año 1998. Arch Bronconeumol 2000; 36: 381-384.

15. De Castro J, Fernández Sánchez JL, Antolín MT, Cordovilla R, Hernández MeZquita MA, González Ruiz JM. Otros factores de riesgo. En: Grupo de estudio CB-SOCALPAR, editores. El carcinoma broncopulmonar en Castilla y León, año 1997. Sociedad Castellano-Leonesa de Patología Respiratoria (SOCALPAR). Bilbao: Cagegraf, 2000. P. $65-80$.

16. Mossman BT, Craighead JE. Mechanisms of asbestos carcinogenesis. Environ Res 1981; 25: 269-280.

17. Mossman BT, Kamp DW, Weitzman SA Mechanisms of carcinogenesis and clinical features of asbestos-associated cancers. Cancer Invest 1996; 14: 466-480.

18. HiRvonen A, SaARikoski ST, LinnainmaA K, Koskinen K, Husgafvel-Pursiainen K, Mattson K et al. Glutathione S-transferase and Nacetyltransferase genotypes and asbestosassociated pulmonary disorders. J Natl Cancer Inst 1996; 88: 1853-1856.

19. Selikoff IJ, Hammond EC, Seidman H. Mortality experience of insulation workers in the United States and Canada, 1943-1976. Ann N Y Acad Sci 1979; 330: 91-116.

20. FinKELSTEIN MM. Mortality among long-term employees of an Ontario asbestos-cement factory. Br J Ind Med 1983; 40: 138-144.

21. Gardner MJ, Powel CA, GaRdNer AW, Winter PD, FLETCHER AC. Continuing high lung cancer mortality among ex-amosite asbestos factory workers and a pilot study of individual anti-smoke advice. J Soc Occup Med 1988; 38: 69-72.

22. Egilman D, REINERT A. Lung cancer and asbestos exposure: asbestosis is not necessary. Am J Ind Med 1996; 30: 398-406.

23. Franceschi S, NAETT C. Trends in smokinf in Europe. Eur J Cancer Prev 1995; 4: 271-284.

24. GonZÁlez CA, Agudo A. Occupational cancer in Spain. Environ Health Perspect 1999; 107 (Suppl 2): 273-277. 
25. Hammond EC, Selikoff IJ, Seidman H. Asbestos exposure, cigarette smoking and death rates. Ann N Y Acad Sci 1979; 330: 473-490.

26. Muscat Je, Stellman SD, Wynder EL. Insulation, asbestos, smoking habits, and lung cancer cell types. Am J Ind Med 1995; 27: 257-269.

27. Rom WN. En: vironmental and occupational medicine, edn. 3. New York, LippincottRaven: 1995.

28. Soutar CA, Simon G, Turner-Warwick M. The radiology of asbestos-induced disease of the lungs. Br J Dis Chest 1974; 68: 235-252.

29. LeE BW, WAIN JC, KeleEy KT, WincKe JK, Christiani DC. Association of cigarette smoking and asbestos exposure with location and histology of lung cancer. Am J Respir Crit Care Med 1988, 157: 748-755.

30. WeIss W. Lobe of origin in the attribution of lung cancer to asbestos. Br J Ind Med 1988, 45: 544-547.

31. Weiss W. Asbestos and lobar site of lung cancer. Occup Environ Med 2000; 57: 358560.

32. BECKET WS. Occupational Respiratory diseases (Rev). N Engl J Med 2000; 342: 406413.

33. ARChER VE, VAGONER JK, LUNDIN FE. Uranium mining and cigarette smoking effects on man. J Occup Med 1973; 15: 204-211.

34. LuBIN JH, BoICE JD. Lung cancer risk from residential radon: metaanalysis of eight epidemiological studies. J Natl Cancer Ins 1997; 89: 49-57.

35. SAMET JM. Indoor radon exposure and lung cancer: risk or not? All over again. J Natl Cancer Inst 1997; 89: 4-6.
36. Martínez C, Rego G. Sílice y cáncer de pulmón. Revisión de la evidencia. Arch Bronconeumol 2002; 38: 33-36.

37. OTt MG, Holder BB, GoRdon HL. Respiratory cancer and occupational exposure to arsenicals. Arch Environ Health 1974; 29: 250-255.

38. LeE-Feldstein A. Cumulative exposure to arsenic and its relationship to respiratory cancer among copper smelter employees. J Occup Med 1986; 28: 296-302.

39. Enterline PE, Marsh GM, Esmen MA, Henderson VL, Callahan CM, Paik M. Some effects of cigarette smoking, arsenic, and SO2 on mortality among US copper smelter workers. J Occup Med 1987; 29: 831-838.

40. TAYLOR PR, QIAO YL, SChatzKin A, YAo SX, Lubin J, MAO BL et al. Relation of arsenic exposure to lung cancer among tin miners in Yunnan Province, China. Br J Ind Med 1989; 46: 881886.

41. Jarup L, Pershagen G, Wall S. Cumulative arsenic exposure and lung cancer in smelter workers: a dose-response study. Am J Ind Med 1989; 15: 31-41.

42. Ferreccio C, González C, Solari J, Noder C. Bronchopulmonary cancer in workers exposed to arsenic: a case- control study. Rev Med Chil 1996; 124: 119-123.

43. Hertz-Picciotto I, Smith AH, Holtzman D, LiPSETT M, AleXEefF G. Synergism between occupational arsenic exposure and smoking in the induction of lung cancer. Epidemiology 1992; 3: 23-31.

44. Bruske-Hohlfeld I, Mohner M, Ahrens W, Pohlabeln H, Heinrich J, Kreuzer M et al. Lung cancer risk in male workers occupationally exposed to diesel motor emissions in Germany. Am J Ind Med 1999; 36: 405-414. 